\section{Un décor de porcelaine est obtenu}

à l'aide de mélanges de pigments

et de composants incolores qui,

en se vitrifiant, fixent l'émail

à la surface de l'objet et révèlent

ainsi la couleur.

Cet article s'intéresse à la stabilité d'un pigment de type spinelle

au cours de la cuisson du décor.

La caractérisation d'un grain

de ce pigment par microscopie

électronique, spectroscopie

optique et absorption des rayons $\mathrm{X}$,

a permis de montrer que

le changement de couleur parfois

observé après cuisson résulte

d'un enrichissement

en chrome de la périphérie

des grains de spinelle.

Les termes en gras suivis d'un astérisque sont définis dans le glossaire, p. 29.

\title{
Couleurs et émaux Des décors de la Manufacture de Sèvres à la réactivité des pigments
}

\author{
Louisiane Verger ${ }^{(1,2)}$ (louisiane.verger@icmcb.cnrs.fr), Olivier Dargaud(1) \\ et Laurent Cormier ${ }^{(2)}$ \\ (1) Cité de la céramique - Sèvres et Limoges, 2 place de la Manufacture, \\ 92310 Sèvres \\ (2) Institut de Minéralogie, de Physique des Matériaux et de Cosmochimie (IMPMC) \\ (Sorbonne Universités, UPMC Univ. Paris 06, CNRS UMR 7590, Muséum national \\ d'Histoire naturelle, IRD UMR 206), 4 place Jussieu, 75005 Paris
}

Un décor de porcelaine est composé d'une ou plusieurs couches partiellement vitrifiées recouvrant la surface de l'objet. Ces décors émaillés auraient été découverts dès 8000 ans avant J.-C., en Égypte ou en Mésopotamie, où des fours de poteries surchauffés auraient donné des céramiques recouvertes d'un fin vernis vitreux, appelé émail (ou glaçure). Outre des propriétés d'imperméabilité, l'émail a ouvert la porte à une effervescence de décorations de la Perse à la Chine, jusqu'à l'essor de la porcelaine en France au $18^{\mathrm{e}}$ siècle avec la Manufacture de Sèvres.

Les décors colorés sont obtenus par mélange d'un composant incolore (fritte de verre ou mélange cristallisé qui va former un verre au cours de la cuisson) avec des phases cristallines contenant les métaux colorants, appelées pigments. Ces derniers étaient initialement des minéraux naturels, mais la quête de nouvelles couleurs et le développement de la chimie au $18^{\mathrm{e}}$ siècle ont conduit à l'élaboration de nouvelles phases cristallines en laboratoire (voir l'encadré, p. 29).

$\mathrm{Au}$ cours de la cuisson pour fixer le décor sur la porcelaine, les grains de pigments et le composant incolore interagissent. La couleur de l'émail après cuisson peut ne pas correspondre à la couleur initiale du pigment. Un tel changement de couleur survient notamment pour des spinelles $^{\star}$ de type gahnite au chrome $\left(\mathrm{ZnAl}_{2-\mathrm{x}} \mathrm{Cr}_{\mathrm{x}} \mathrm{O}_{4}\right)$, interdisant leur utilisa- tion dans certaines conditions de cuisson et dans certains décors, alors que la teinte reste parfaitement inaltérée dans d'autres. $\mathrm{Au}$ cours de la cuisson à $1280^{\circ} \mathrm{C}$ d'une des palettes principales de la bibliothèque de décors de la manufacture, la couleur du pigment, initialement rose, s'altère et une teinte marron est observée. La spectroscopie d'absorption optique montre alors que la cuisson a induit un décalage des bandes d'absorption principales de l'élément chromophore, $\mathrm{Cr}^{3+}$, vers les plus faibles nombres d'onde (fig. 1a). Ces changements soulignent une réactivité des pigments, souvent mal comprise, et contrôlée empiriquement dans les procédés industriels et artisanaux.

\section{Une microstructure complexe de l'émail}

Les décors étudiés ont été préparés spécialement pour cette étude à partir d'un pigment composé majoritairement d'un spinelle $\mathrm{ZnAl}_{1.59} \mathrm{Cr}_{0.41} \mathrm{O}_{4}$, de couleur rose. Une seule couche colorée est appliquée au pinceau sur des supports ronds.

L'analyse d'une coupe transversale par microscopie électronique à balayage (MEB) révèle la stratigraphie du décor (fig. 1b). Le support en porcelaine (en bas de la photo $1 \mathrm{~b}$ ) se distingue de la couche de décor coloré au-dessus. L'émail est hétérogène, composé de bulles (zones noires) et de grains de pigment (zones grises), inclus dans une matrice amorphe résultant de la vitrification du composant 


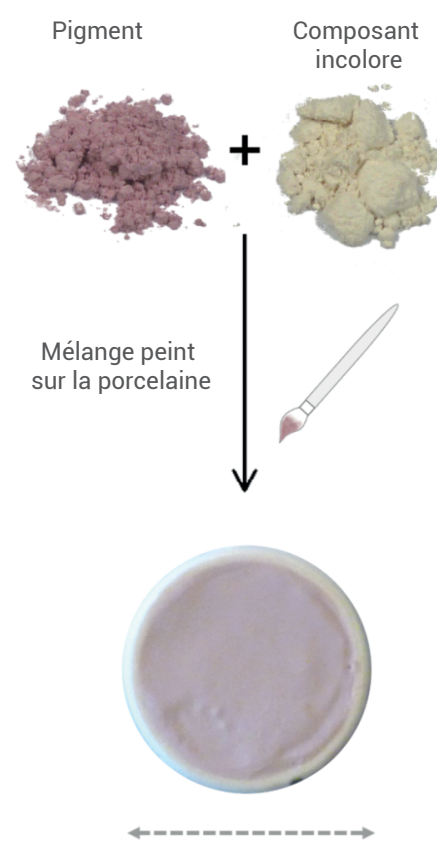

$20 \mathrm{~mm}$

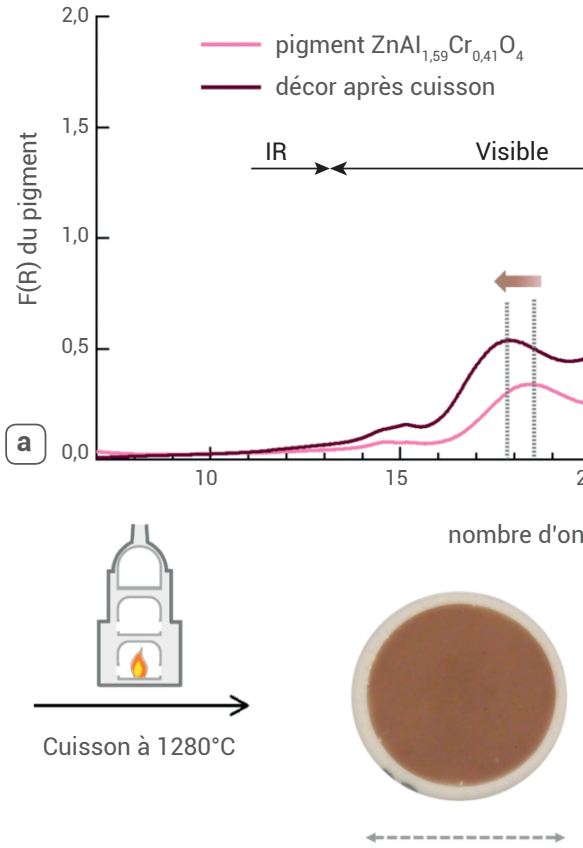

$17 \mathrm{~mm}$
Si

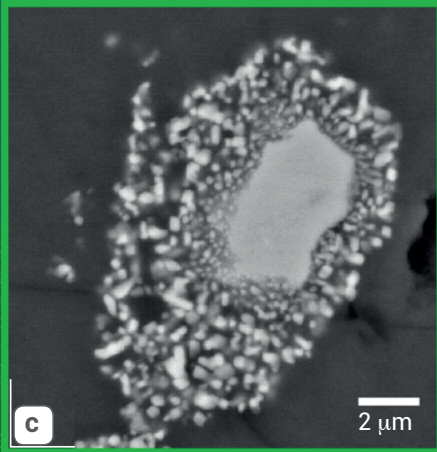

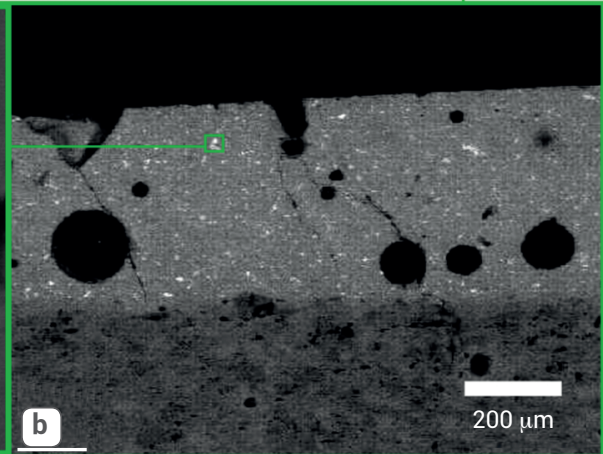

1. Préparation et analyses des échantillons de décors de porcelaine. Le mélange entre un pigment et un composant incolore est appliqué au pinceau avec un liant organique sur des supports en porcelaine, qui sont ensuite cuits à $1280^{\circ} \mathrm{C}$.

(a) Fonctions de rémission* calculées à partir des spectres de réflectance diffuse du pigment de départ et du décor après cuisson, obtenus dans le domaine visible $\left(\approx 13300\right.$ à $\left.26300 \mathrm{~cm}^{-1}\right)$ et proche UV $\left(\geq 26300 \mathrm{~cm}^{-1}\right)$. On observe après la cuisson un déplacement vers les faibles nombres d'onde des deux bandes d'absorption situées initialement vers 18500 et $26000 \mathrm{~cm}^{-1}$.

(b-c) Analyses au microscope électronique à balayage par électrons rétrodiffusés d'une coupe transversale (b) et d'un grain de pigment (c) après cuisson.

(d) Cartographies par EDX* (analyse dispersive en énergie des rayons X) des principaux éléments chimiques composant le décor dans la zone du grain observé sur la figure $1 \mathrm{c}$.

incolore. La figure 1c montre un grain spécifique, cristallisé, partiellement altéré en périphérie et représentatif des autres grains. Le centre du grain apparait compact, alors que la périphérie est fragmentée en plusieurs cristaux. Cette hétérogénéité de morphologie correspond à une différence de composition chimique : le cœur du grain est riche en aluminium et en zinc, alors que la périphérie est riche en chrome (fig. 1d).
Le développement de la couche d'altération à la surface du pigment est sans doute à l'origine du changement de couleur après cuisson : est-ce que cette couche est encore du spinelle ou une nouvelle phase cristalline ? Est-ce que l'environnement local du chrome est modifié entre le centre et la périphérie du pigment ? Pour répondre à ces questions, il est nécessaire de déterminer cet environnement du chrome à l'échelle locale.

\section{Environnement local du chrome dans l'émail}

Des mesures d'absorption des rayons $\mathrm{X}$ au voisinage du seuil $\left(\right.$ XANES $\left.^{\star}\right)$ permettent d'obtenir des informations sur l'environnement local du chrome, que cet environnement soit cristallisé ou vitreux. Un faisceau microfocalisé disponible sur la ligne ID21 de l'ESRF [1] offre une résolution spatiale submicrométrique, 
>>

permettant de sonder préférentiellement le centre ou la périphérie du grain (fig. 2).

L'encart (c) de la figure 2 correspond à la cartographie par fluorescence des rayons $\mathrm{X}$ acquise sur le grain de pigment observé figure $1 \mathrm{c}$.

Les trois structures $a, b$, et $c$, observées sur la figure $2 \mathrm{a}$, sont caractéristiques de l'ion $\mathrm{Cr}^{3+}$ en symétrie octaédrique dans les spinelles. Les spectres XANES du pigment initial et du centre du grain sont semblables. En revanche, de légères différences sont observées entre le centre du grain et la périphérie, comme le pic $c$ décalé de $0,92 \mathrm{eV}$ vers les plus faibles énergies. La région du préseuil (encart (b) de la figure 2) montre des variations plus importantes : deux structures $(\alpha$ et $\beta$ ) sont communes aux deux zones et une troisième structure $(\gamma)$ est uniquement observée sur le spectre de la périphérie du grain.

Ces changements (décalage du pic $c$ et apparition de la structure $\gamma$ ) sont par ailleurs observés quand la teneur en chrome augmente le long de la solution solide $\mathrm{ZnAl}_{2-\mathrm{x}} \mathrm{Cr}_{\mathrm{x}} \mathrm{O}_{4}$ [2]. La phase en périphérie du grain de pigment est donc constituée d'un spinelle de même structure que le pigment initial, mais avec une teneur en chrome plus importante, ce qui est confirmé par la cartographie de fluorescence (encart (c) de la figure 2) et par analyse Rietveld ${ }^{\star}$ des spectres de diffraction des rayons $\mathrm{X}$.

La présence de cette phase enrichie en chrome explique le changement de couleur du décor. En effet, la couleur des spinelles varie avec la teneur en chrome le long de la solution solide $\mathrm{ZnAl}_{2-\mathrm{x}} \mathrm{Cr}_{\mathrm{x}} \mathrm{O}_{4}$. La teinte rose commence par s'intensifier, puis une nuance marron apparait pour $x=1$ jusqu'à la zincochromite verte, $\mathrm{ZnCr}_{2} \mathrm{O}_{4}$. L'environnement local autour de l'ion chrome (distances $\mathrm{Cr}-\mathrm{O}$ et/ou coordinence) est faiblement affecté dans la solution solide, comme montré par des calculs DFT ${ }^{\star}$ (Density Functional Theory) des spectres XANES [2]. Au contraire, ces calculs suggèrent que la proximité de seconds voisins chrome modifie le champ cristallin de l'ion $\mathrm{Cr}^{3+}$ et donc la couleur des spinelles. L'analyse des spectres XANES révèle ainsi une évolution de l'environnement du chrome, indiquant un double mécanisme de dissolution puis de stabilisation d'un nouveau spinelle enrichi en chrome, conduisant à une couleur différente.

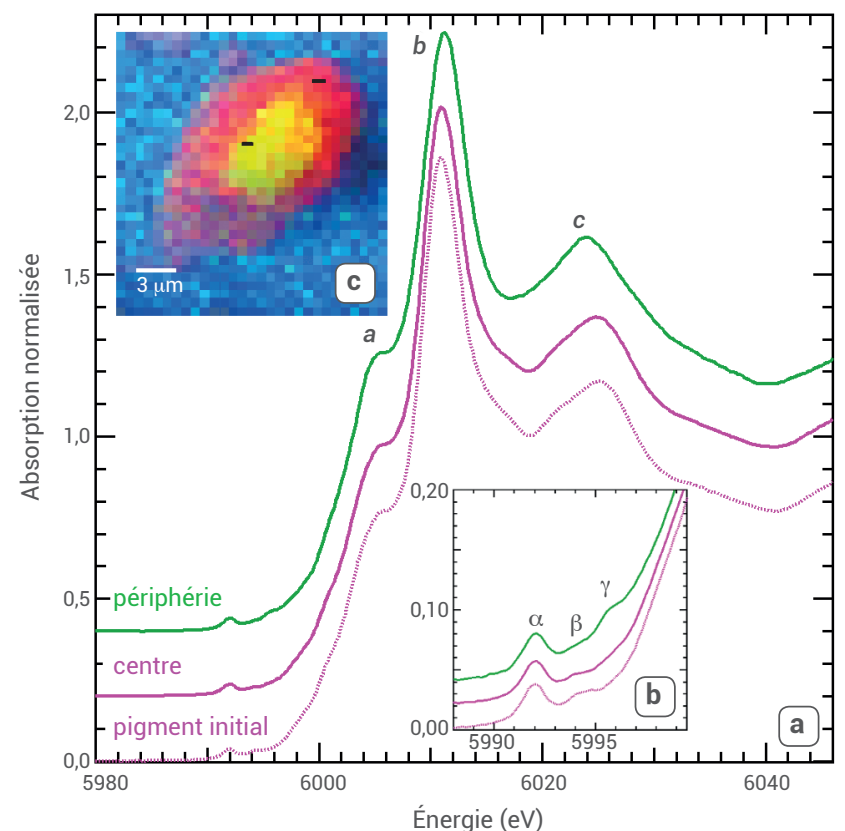

2. Analyse par spectroscopie d'absorption des rayons $X$ du décor de porcelaine.

(a) Spectres XANES au seuil K du Cr du pigment de départ (rose pointillé), d'une zone au centre (rose) et d'une zone en périphérie d'un grain (vert). (Pour plus de clarté, les courbes sont décalées en ordonnée).

(b) Zoom sur la région du préseuil.

(c) Cartographie de fluorescence des rayons X des principaux éléments : Al (vert), Si (bleu) et $\mathrm{Cr}$ (rouge), acquise sur le grain de pigment de la figure 1c. Les rectangles noirs représentent les points d'analyse XANES, et leur taille correspond à la résolution spatiale du faisceau de rayons $X\left(0,7 \times 0,3 \mu \mathrm{m}^{2}\right)$.

L'enrichissement en chrome de la phase spinelle dans la périphérie du grain est en fait le résultat de la diminution des teneurs en aluminium et en zinc, qui diffusent depuis le spinelle vers le verre [3]. En modifiant la composition du pigment et en ajoutant de l'oxyde de zinc $\mathrm{ZnO}$ au composant incolore, on a pu faire cristalliser et stabiliser la phase spinelle, permettant son utilisation dans cette palette de décor. La compréhension du mécanisme d'altération illustre ainsi l'impact direct de ces recherches sur les procédés.

L'empirisme avait consacré certaines utilisations bien particulières dans des conditions très contraintes en termes cinétique et thermodynamique ou de compositions. L'étude de ces phénomènes dans leurs réussites et leurs limites permet de mieux comprendre à la fois les conditions historiques de production, mais aussi et surtout de proposer de nouvelles voies de développement au regard des possibilités apportées par les outils de la science contemporaine.

\section{Références}

1 M. Motte et al., "The ID21 X-ray and infrared microscopy beamline at the ESRF: status and recent applications to artistic materials", J. Anal. At. Spectrom. 32 (2017) 477-493.

2- L. Verger et al., "Spectroscopic properties of $\mathrm{Cr}^{3+}$ in the spinel solid solution $\mathrm{ZnAl}_{2-x} \mathrm{Cr}_{x} \mathrm{O}_{4}$ ", Physics and Chemistry of Minerals 43 (2016) 33-42.

3. L. Verger et al., "Interaction between $\mathrm{Cr}$-bearing pigments and transparent glaze: A transmission electron microscopy study", Journal of Non-Crystalline Solids 459 (2017) 184-191. 


\section{Le chrome et la synthèse des pigments à la Manufacture de Sèvres}

La finesse et les couleurs des décors des porcelaines de la Manufacture de Sèvres concourent à faire sa renommée, depuis sa création en 1740 . Toutes les étapes de la production des porcelaines sont menées au sein de la Manufacture. Le laboratoire est en charge de la synthèse des pigments.

L'élément chrome a été découvert en 1797 par Nicolas Louis Vauquelin, et introduit seulement quelques années plus tard dans les procédés de la Manufacture par Alexandre Brongniard.

Au cours du $19^{e}$ siècle, les recherches à la Manufacture sur la synthèse des minéraux s'intensifient afin d'obtenir des poudres colorées pour les décors de porcelaine, amenant Jacques-Joseph Ebelmen à découvrir la croissance cristalline par la méthode des flux en 1847. Les minéraux composés de chrome, dont principalement les spinelles comme la gahnite au chrome $\left(\mathrm{ZnAl}_{2-\mathrm{x}} \mathrm{Cr}_{\mathrm{x}} \mathrm{O}_{4}\right)$, font l'objet d'un intérêt particulier, en raison de la riche palette de couleurs qu'ils offrent (fig. E1).

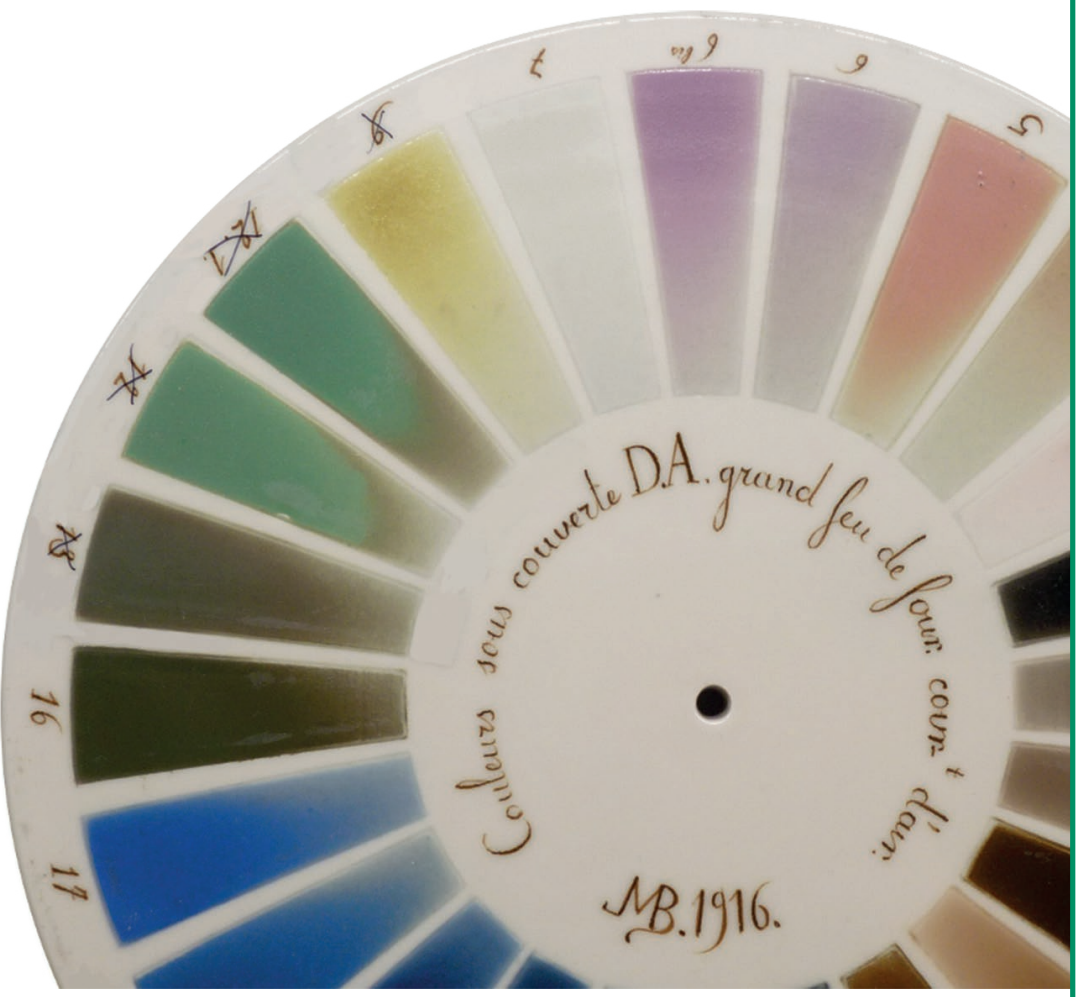

E1. Exemple de palette de décors de la Manufacture de Sèvres.

\section{: Glossaire}

Minéraux cristallins, de formule générale $\mathrm{AB}_{2} \mathrm{O}_{4}$ et de structure cubique à faces centrées,
où $\mathrm{A}$ et $\mathrm{B}$ sont des ions métalliques et dont le composé prototype $\mathrm{es}^{2} \mathrm{O}_{4}$.

Fonction de rémission d'un spectre de réflectance

EDX

(Energy Dispersive

X-ray Spectrometry)

XANES

(X-ray Absorption

Near Edge Structure)

Analyse de Rietveld

DFT

(Density Functional Theory)
Fonction calculée à partir du spectre de réflectance diffuse, qui correspond (à un facteur de translation près) au spectre d'absorption du matériau obtenu par transmission.

Technique utilisée pour l'analyse chimique élémentaire d'un matériau excité par un faisceau de particules chargées ou de photons, et dont le spectre d'émission de rayons $\mathrm{X}$ montre des pics caractéristiques des éléments contenus.

Méthode d'analyse spectroscopique, qui utilise l'absorption des rayons $\mathrm{X}$ par un matériau au voisinage ( $\simeq 50 \mathrm{eV}$ ) d'un seuil (préseuil et montée du seuil). Elle permet d'obtenir des informations structurelles et électroniques sur le matériau étudié.

Méthode d'analyse des diagrammes de diffraction de rayons $X$ et de neutrons sur poudres, qui ajuste par moindres carrés un profil calculé au spectre expérimental complet.

Méthode de calcul quantique de la structure électronique, utilisée en particulier en matière condensée, où l'on remplace la fonction d'onde multiélectronique par la densité électronique en tant que quantité de base pour les calculs. 